\title{
AHP method to determine the optimal technological variant for maxillofacial implant fabrication
}

\author{
Luciana Laura Dincă*, Alexandra Banu, Aurelian Vișan and Nicolae Ionescu \\ Politehnica University of Bucharest, Department of Manufacturing Technology, Splaiul \\ Independenţei no. 313, sector 6, Bucharest, RO-060042, Romania
}

\begin{abstract}
The paper describes different technologies for manufacturing maxillofacial implant. By using the AHP method the AM, the direct manufacturing technology SLM is the optimal process. Technological variants for obtaining a maxillofacial implant that replaces the right zygomatic bone and a part of maxilla, based on a real clinical case are presented. Starting from the possibility of processing the biocompatible metallic materials used in the oral and maxillofacial technique, it is possible to classify the technological process variants in procedures that use and do not use additive manufacturing technologies. The aim of this paper is to show how important it is to select the optimal technological process in obtaining a maxillofacial implant. It has been analyzed the technological processes of achieving the maxillofacial implant, both those which use the additive manufacturing technologies and those which do not use such technologies, highlighting not only the benefits but also their limits. Based on criteria such as: mechanical strength, porosity, roughness, accuracy, anchorage, time and cost, by using the AHP method, it is possible to choose the optimal technological process for achieving the maxillofacial implant.
\end{abstract}

\section{Introduction}

The purpose of using maxillofacial implants is to restore the appearance and functionality of the affected part, which will help to improve the emotional state of the patients.

Because each patient is unique, therefore anatomically different, to manufacture an implant has always been a big problem, that has been solved by producing customized implants, to fit each case, that use rapid prototyping technologies [1].

The advances in the design, production and implantation of maxillofacial prostheses are strictly related to the development of Computerized Tomography (CT), Magnetic Resonance (MRI), Computer Aided Design (CAD), Computer Aided Manufacturing (CAM), and of Rapid Prototyping technologies (RP).

Starting from the possibility of processing the biocompatible metallic materials used in the maxillofacial field, it is possible to classify the variants of technological processes in procedures that use and that do not use rapid prototyping technologies.

\footnotetext{
*Corresponding author: lucianalaura_shamieh@yahoo.com
} 
Titanium as an implanted material used for the maxillofacial reconstruction, has started to be used after 1967 and has become a choice in maxillofacial field thanks to its biological and mechanical properties, being able to support the bone growth and thus osseointegration to occur [2]. In the maxillofacial applications both, material used to obtain the implant with high biocompatibility and specific characteristics and the technology used for producing the implants to meet high quality standards are very important.

The advance of Additive Manufacturing systems has provided a route for the fabrication of customized implants based on a 3D model, allowing the manufacture of complex and internal detailed parts because of their methodologies of building the object by adding material, layer upon layer [3].

Opposite to the Additive Manufacturing (AM) technologies which make the object by adding material, layer-by-layer, directly from a digital model, that does not require the use of tools, the conventional or subtraction manufacturing processes such as milling, are used to create a part or product by cutting away material into a desired final shape and size, involving large waste material. Also, the conventional manufacture of medical implants by casting or milling presents the disadvantage of a significant limitation of their geometry.

Compared with conventional techniques, the use of additive manufacturing technologies leads to more accurate and faster surgeries [4].

In the maxillofacial field, Computer - Aided Surgery (CAS) procedures comparing to conventional techniques has improved the results in obtaining customized implants [5].

The most commonly additive manufacturing technologies used in the production of enduse implants are: Selective Laser Sintering (SLS), Selective Laser Melting (SLM) and Electron Beam Melting (EBM). Thanks to the possibility to obtain a perfect implant from the patient data, additive manufacturing technologies have acquired a special place in maxillofacial reconstruction [6].

As a result of research that were realized around the world, including the Politehnica University of Bucharest [7], and universities from Ireland [8], in the case of a maxillofacial implant it is possible to find several technological process variants.

There have been analyzed the technological processes for achieving maxillofacial implants, both those that use the additive technologies and those that do not use such technologies, highlighting not only the benefits but also the limitations of these technologies. Currently, the additive manufacturing technologies are used in the technological process of obtaining a maxillofacial implant, both for the fabrication of the mold that helps to achieve the implant and for the direct realization of the implant starting from the powder material in raw state.

The main objectives of the presented paper are to highlight the importance of choosing the optimal technological process in the maxillofacial implant case that will replace the right zygomatic bone and a part of maxilla which were removed after maxillofacial cancer and to identify the application of the Analytic Hierarchy Process (AHP) method in the selection of an optimal technological process.

\section{Experimental details}

\subsection{Materials}

In order to determine the optimal technological process, there were selected the technological variants for obtaining the maxillofacial implant that will replace the right zygomatic bone and a part of maxilla, starting from the medical images of the case study. By choosing titanium as an implanted material used for the maxillofacial reconstruction, it was considered 
the possibility of processing it, and there were classified the technological processes into two big categories namely which does and does not use additive manufacturing technologies.

\subsection{Method}

In order to obtain the optimal technological process there has been considered the AHP analysis method. Based on criteria such as: mechanical strength, accuracy, anchorage, time and cost, by using the AHP method, the optimal technological process for achieving the maxillofacial implant will be selected.

Introduced in 1980 by Thomas Saaty, the Analytical Hierarchy Process (AHP) is an effective tool that can be used to set priorities and make the best decisions.

It is possible to be distinguished four stages of the AHP method application: a) Problem structuring. This is the most important part of decision-making and fundamental to the process of the AHP, because the problem is broken down into a hierarchy of goal, criteria, sub-criteria and alternatives; b) Data collection: Data are collected from experts, the comparisons are made for each criterion and can be evaluated as 1-equally important, 3 moderately important, 5 - strictly more important, 7 - very strict, 9-extreme importance; and 2, 4, 6, 8 - intermediate values. c) Determination of the relative weight: The pairwise comparisons of various criteria generated at step 2 are organized into a square matrix where the diagonal elements of the matrix are 1; d) The final result - solution of the problem -The AHP produces weight values for each alternative based on the judged importance of one alternative over another with respect to a common criterion. By respecting each criterion, the rating of each alternative is multiplied by the weights of the sub-criteria and aggregated to get local ratings, which are multiplied by the weights of the criteria and aggregated to get global ratings. The ultimate value is used to make a decision about the problem [8].

\section{Results and discussions}

\subsection{Establishing the technological variants}

This study started from the digital images of the patient affected by maxillofacial cancer, which caused the removal of the zygomatic bone and a part of the maxilla. The images processing and 3D reconstruction were done using the MIMICS software tool that allows the conversion of DICOM images into a 3D model (see Figure 1).



Fig. 1. The facial morphology-the reconstruction model.

By having the facial morphology of the case study, and by following the researches carried out in this field [7], four technological variants have been considered possible for realizing the implant.

A. Al-Ahmari, et.al. (2015) study presents a mandibular reconstruction that was realized by using additive manufacturing technologies and have shown that technological process 
which directly generates the physical implant using additive manufacturing, has been successfully applied [4].

In the planning phase of the fabrication of the implant, it is projected that the implant has complex and inclined structures, porous structure and roughness to ensure the anchoring, high accuracy and weight as near as possible to the anatomical bones. The technological variants considered for the manufacturing of the maxillofacial implant from titanium material were classified in four variants, as follows: a process that does not use AM, the implant being obtained by 3D milling; technological processes that use AM in such a way: the use of AM to obtain the model that will helps shell molding, the use of AM to obtain the casting mold, and AM used to obtain the implant directly. The four types of technological process are presented in Figure 2.

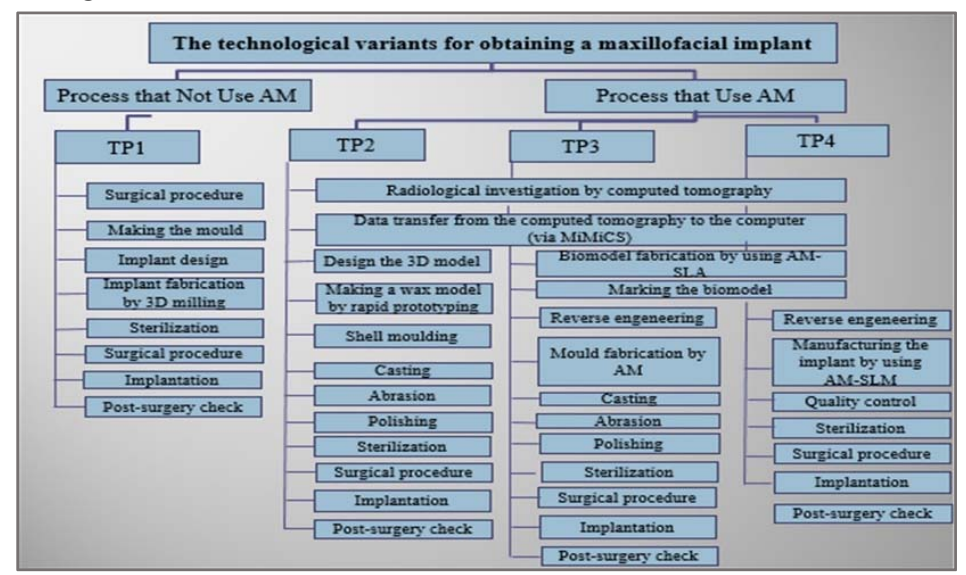

Fig. 2. The technological variants for obtaining the maxillofacial implant.

\subsection{The steps for obtaining the optimal technological process.}

In this study the all technological variants considered for the obtaining of a maxillofacial implant were analyzed by using the AHP method.

For evaluating the process, five criteria were taken into consideration:

C1- mechanical strength;

C2- accuracy;

C3- anchoring;

C4- time;

C5-cost.

The first step was to build a hierarchy for the decision so, the problem has been defined and presented in Figure 3.

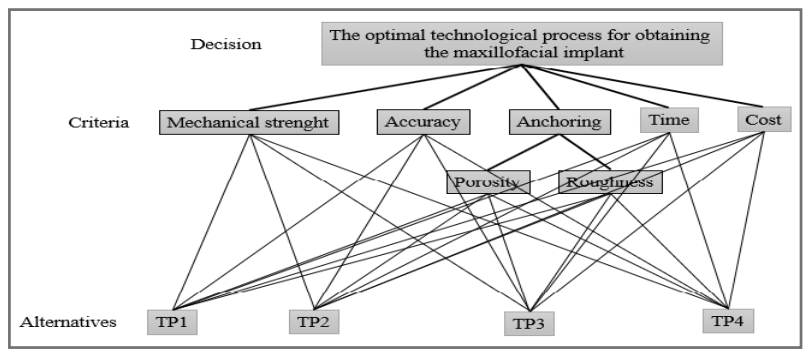

Fig. 3. Structuring the problem for the selection of the optimal technological process in the case of the maxillofacial implant. 
The structuring problem is very important step and make it is possible to better understand the decision of obtaining the optimal technological process to be achieved, the criteria to be used, and the alternatives to be estimated. For an easier method application, DecisorGui software [9] was used. Each of the five criteria has been compared against each other (pairwise) and the results are presented in Figure 4.

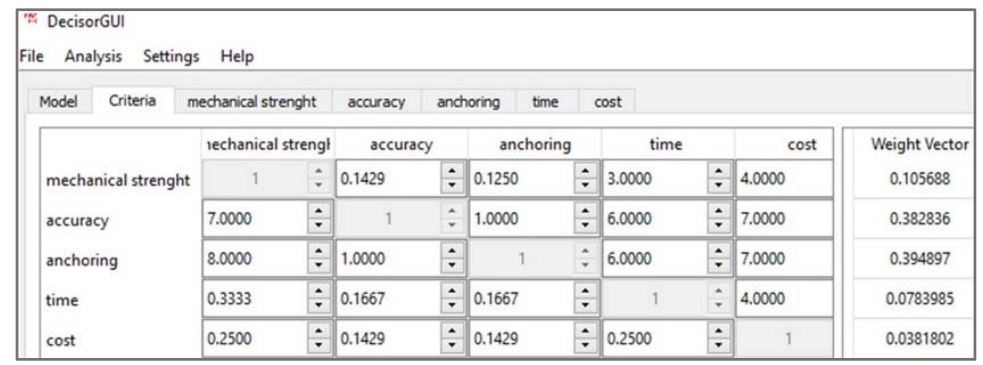

Fig. 4. The criteria importance.

The evaluation of the variants, with respect to the five criteria taken into consideration are presented in Figure 5.

\begin{tabular}{|c|c|c|c|c|c|c|c|c|c|c|c|c|c|c|c|c|c|c|c|c|c|c|}
\hline \multirow{3}{*}{\begin{tabular}{|c|} 
Model \\
TP1 \\
\end{tabular}} & \multirow{2}{*}{\multicolumn{2}{|c|}{$\begin{array}{c}\text { Criteria } \square \text { mec } \\
\text { TP1 }\end{array}$}} & \multicolumn{2}{|c|}{ nechanical strenght } & \multicolumn{2}{|c|}{ accuracy } & \multicolumn{2}{|c|}{ anchoring } & \multirow{2}{*}{\multicolumn{2}{|c|}{$\begin{array}{l}\text { time } \\
\text { TP4 }\end{array}$}} & & \multirow{3}{*}{\begin{tabular}{l|l} 
& Mode \\
or & \\
TP1
\end{tabular}} & \multicolumn{2}{|c|}{ Criteria } & \multicolumn{3}{|c|}{ mechanical strenght } & \multirow{2}{*}{\multicolumn{2}{|c|}{$\begin{array}{c}\text { accuracy ancho } \\
\text { TP3 }\end{array}$}} & \multirow{2}{*}{\multicolumn{2}{|c|}{$\begin{array}{l}\text { time } \\
\text { TP4 }\end{array}$}} & \\
\hline & & & TP2 & & \multicolumn{3}{|c|}{ TP3 } & & & & Weight Vector & & & \multicolumn{2}{|l|}{ TP1 } & \multicolumn{2}{|c|}{ TP2 } & & & & & \multirow{2}{*}{$\begin{array}{c}\text { Weight Vector } \\
0.416803\end{array}$} \\
\hline & 1 & $:$ & 2.0000 & $:$ & 3.0 & 0000 & $:$ & \multicolumn{2}{|c|}{4.0000} & & 0.471498 & & & 1 & $:$ & 2.0000 & $:$ & 2.0000 & $:$ & \multicolumn{2}{|c|}{3.0000} & \\
\hline TP2 & 0.5000 & $\div$ & 1 & $:$ & 2.0 & 0000 & $:$ & & .0000 & $:$ & 0.25498 & TP2 & \multicolumn{2}{|l|}{0.5000} & $:$ & 1 & $:$ & 2.0000 & $:$ & \multicolumn{2}{|l|}{2.0000} & 0.269481 \\
\hline \multirow{2}{*}{$\begin{array}{l}\text { TP3 } \\
\text { TP4 }\end{array}$} & 0.3333 & $\div$ & 0.5000 & $\div$ & & 1 & $:$ & & 0000 & $:$ & 0.165264 & \multirow{2}{*}{$\mid \begin{array}{l}\text { TP2 } \\
\text { TP3 } \\
\text { TP4 }\end{array}$} & \multicolumn{2}{|l|}{0.5000} & $:$ & 0.5000 & $:$ & 1 & : & \multicolumn{2}{|l|}{2.0000} & 0.192777 \\
\hline & 0.2500 & $\div$ & 0.5000 & $\div$ & 0.5 & 5000 & $\because$ & & 1 & $:$ & 0.108259 & & \multicolumn{2}{|c|}{0.3333} & $\div$ & 0.5000 & $:$ & 0.5000 & $\div$ & 1 & $:$ & 0.120938 \\
\hline Model & Criteria & mech & chanical streng & & accu & uracy & anchor & & time & cost & & Mode & Crit & & & chanical stren & & accuracy & ancho & oring $\overline{t \text { tin }}$ & $\cos$ & \\
\hline & TP1 & & TP2 & & & TP3 & & & TP4 & & Weight Vector & & & TP1 & & TP2 & & $\mathrm{T}$ & & $T$ & & Weight Vector \\
\hline TP1 & 1 & $:$ & 0.1429 & $:$ & 0.16 & 667 & $\div$ & 0.11 & & : & 0.0407714 & TP1 & 1 & & $:$ & 0.1250 & $\div$ & 0.3333 & $:$ & 0.1429 & $:$ & 0.0516343 \\
\hline TP2 2 & 7.0000 & $:$ & 1 & $:$ & 2.00 & & $\div$ & 0.33 & 333 & $:$ & 0.248876 & TP2 & 8.0000 & & $:$ & 1 & : & 3.0000 & $:$ & 0.5000 & $:$ & 0.343079 \\
\hline TP3 & 5.0000 & $:$ & 0.5000 & $\div$ & & 1 & : & 0.25 & & $:$ & 0.16391 & TP3 & 3.0000 & & $\div$ & 0.3333 & $\div$ & 1 & $:$ & 0.3333 & $\div$ & 0.139823 \\
\hline TP4 & 3.0000 & $:$ & 3.0000 & $:$ & 4.00 & 000 & $\div$ & & 1 & : & 0.546442 & TP4 & 7.0000 & & $\because$ & 2.0000 & $\div$ & 3.0000 & : & 1 & : & 0.465464 \\
\hline & & & & & Model & Criterit & & & chanical str & & accuracy & nchorin & time & 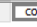 & cost & & & & & & & \\
\hline & & & & & & TP & P1 & & TP & & TP3 & & $\mathrm{TP}$ & & & Weight Ve & & & & & & \\
\hline & & & & & TP1 & 1 & & $:$ & 0.1111 & $\div$ & 0.2000 & $: 0$ & 0.1429 & $\div$ & & 0.04275 & & & & & & \\
\hline & & & & & TP2 & 9.0000 & & $\div$ & 1 & : & 4.0000 & $: 0$ & 0.3333 & $:$ & & 0.34065 & & & & & & \\
\hline & & & & & TP3 & 5.0000 & & $\div$ & 0.2500 & $:$ & 1 & $: 0$ & 0.5000 & $:$ & & 0.16912 & & & & & & \\
\hline & & & & & TP4 & 7.0000 & & $\div$ & 3.0000 & $:$ & 2.0000 & : & 1 & : & & 0.4474 & & & & & & \\
\hline
\end{tabular}

Fig. 5. The evaluation of the variants for each criterion.

By using the AHP method there were found the following results as presented in Figure 6.

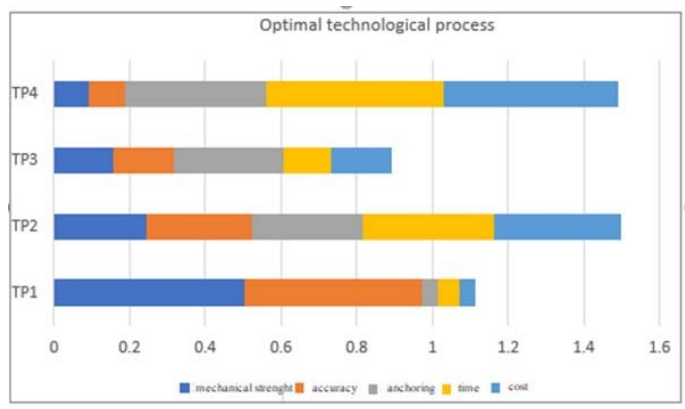

Fig. 6. The optimal alternative selection. 
In the case of the maxillofacial implant, that will replace the zygomatic bone and a part of maxilla, the TP4 is the optimal technological process because it is able to realize the complex shape of the implant, with inclined structures which are impossible to be realized by using conventional methods. This method is also able to produce the porous structure that allows further on the tissue ingrowth, as well as an implant.

\section{Conclusions}

Based on the analysis made in this paper, it was found that TP4, the direct fabrication of the implant using additive manufacturing technology SLM, is the optimal technological process in the case of maxillofacial implant that will replace the zygomatic bone and a part of the maxilla. In the application of the AHP method, the fourth alternative, has resulted in obtaining the highest total value. In order to obtain the customized implant, starting from the patient data, an accurate implant with complex shape and inclined structures, with porous structure and roughness to ensure the anchoring, and weight as near as possible to the anatomical bones, with a high durability, the additive manufacturing technologies are the best choice. Besides the lack of constraints, the customized implants obtained by additive manufacturing technologies present a lot advantages, such as: planning and simulation before surgery, shortening of the anesthesia time, low patient trauma and shortening of the recovery time of the patient this issue being favorable for both the patient and the surgeon, leading to a better medical result and so the success of the surgery at the end.

As a conclusion it is possible to state that the importance of choosing the optimal technological process comes from the possibility of obtaining the perfect implant, customized on medical case, and by considering the fact that additive manufacturing technologies have a lot of benefits but also do have its limitations, so they are the best to be used in medical applications, for obtaining complex geometries with porous structures as similar as possible to the anatomical structures.

\section{References}

1. A.L. Jardinia, M.A. Larosa, C. Amélia de Carvalho Zavaglia, L.F. Bernardesa, C.S. Lamberta, P. Kharmandayana, D. Calderonia, and R.M. Filhoa, Virt. and Phys. Prot 9, 2, 115-125 (2014)

2. S. Nayar, S. Bhuminathan, W.M. Bhat, J .Pharm Bioallied Sci. 7, 1, S216-S219 (2015)

3. A. Al-Ahmari, E.A. Nasr, K. Moiduddin, M. Alkindi, and A. Kamrani, Proceedings of the 2015 Int. Conf. on Ind. Eng. and Operat. Manag. Dubai, UAE, March 3-5 (2015)

4. D. Serban, D. Boyle, S. Lohfeld, P. McHugh, N. Peckitt, Comp. Ass. Rad. and Surgery 17th International Congress and Exhibition, London, UK, June 25-28 (2003)

5. L.L. Dincă, A. Banu, A. Vişan, (MATEC Web of Conferences 137, 02001, 2017)

6. D. Șerban, Contributions to research - Development maxillofacial prosthesis, $\mathrm{PhD}$ Thesis (Politehnica University of Bucharest, 2015)

7. S. Lohfeld, P. McHugh, D. Serban, D. Boyle, G. O’Donnell, N. Peckitt, J. of Mat. Process. Techn. 189, 2-3, (2006)

8. D. Zoran, M. Saša, P. Dragi, Underground Mining Engineering, 19 (2011)

9. https://github.com/unisc/decisorGUI 